\title{
BiBLIOGRAPHY
}

1. M. Heins, Algebraic structure and conformal mapping, Technical Report issued by the Institute for Advanced Study, 1957.

2. H. L. Royden, Rings of analytic and meromorphic functions, Trans. Amer. Math. Soc. vol. 83 (1956) pp. 269-276.

3. W. Rudin, Some theorems on bounded analytic functions, Trans. Amer. Math. Soc. vol. 78 (1955) pp. 333-342.

4. H. Weyl, Die Idee der Riemannschen Flaechen, Berlin, 1923.

STANFORD UNIVERSITY

\section{A SEMI-SIMPLE MATRIX GROUP IS OF TYPE $I$}

\section{W. FORREST STINESPRING}

The purpose of the following note is to give a short simple proof of the most important special case of Harish-Chandra's result [3, Theorem 7] that every connected semi-simple Lie group is of type $I$. We shall prove that every continuous unitary representation of a connected semi-simple matrix group is of type $I$. This fact is a consequence of theorems in two papers of Godement [1, Theorem 2; 2 , Theorem 8]. An improved version of Godement's method is used here. The improvements are:

(1) the argument is considerably shorter;

(2) infinite dimensional nonunitary representations are not needed;

(3) the argument is completely global; no direct integrals are used. These improvements seem not to have been noticed before.

Definition. We shall say that an algebra is of type $I_{s n}$ if it satisfies the identities

$$
\left[A_{1}, \cdots, A_{r}\right]=\sum_{i_{1}, \cdots, i_{r}} \operatorname{sgn}\left(\begin{array}{c}
1 \cdots r \\
i_{1} \cdots i_{r}
\end{array}\right) A_{i_{1}} A_{i_{2}} \cdots A_{i_{r}}=0
$$

for all $r$ for which the algebra of all $n \times n$ matrices satisfies them.

In [5, §2], Kaplansky shows that the algebra of all $n \times n$ matrices is of type $I_{\leqq n}$ but not of type $I_{\leqq n-1}$. It follows from the definition that an algebra is of type $I_{\Xi n}$ if it is a subalgebra of an algebra of type $I_{\leqq n}$, or if it is a homomorphic image of an algebra of type $I_{\unlhd n}$, or if it has a separating family of homomorphisms into algebras of type $I_{\leqq n}$. Since the above identities are linear in each variable, a von Neumann algebra is of type $I_{\leqq n}$ if it has a weakly dense subalgebra of type $I_{\leqq n}$. A von Neumann algebra of type $I$ (in the usual sense) is

Received by the editors May 5, 1958. 
the direct sum of von Neumann algebras of homogeneous type $I_{k}$ where $k$ runs over the cardinals. Since an algebra of type $I_{\leqq n}$ in the sense of the above definition has no subalgebras isomorphic to the $(n+1) \times(n+1)$ matrices, it is clear that a von Neumann algebra is of type $I_{\leqq n}$ as defined above if and only if it is of type $I$ in the usual sense and all the direct summands of homogeneous type $I_{k}$ are zero for $k>n$. Consequently the present terminology accords with the usual one.

TheORem 1. Let $G$ be a connected semi-simple matrix group. Let $L$ be the left regular representation of $G$ on $L_{2}(G)$, and let $\&$ be the von Neumann algebra generated by $L$. Let $K$ be a maximal compact subgroup of $G$, and let $\rho$ be an irreducible representation of $K$ of degree $n$ with character $\bar{\chi} / n$. Let $E$ be the projection given by

$$
E=\int_{K} \chi(k) L(k) d_{K} k
$$

where $d_{K} k$ is Haar measure on $K$ and the integral is in the weak sense. Then the algebra $E \& E$ is of type $I_{\unlhd} n^{2}$.

Proof. The group $G$ has a connected solvable subgroup $S$ such that $G=K S$ (see [4, Lemma 3.11 and the proof of Lemma 3.12]). Let $a$ be the algebra of all $L_{\phi}$ with $\phi$ in $C_{0}(G)$ where $L_{\phi}=\int_{G} \phi(x) L(x) d x$. It is sufficient to show that $E Q E$ is of type $I_{\leqq} n^{2}$. This will be done by exhibiting a separating family of homomorphisms of $E Q E$ into $q \times q$ matrices with $q \leqq n^{2}$. Such a family is provided by the finite dimensional irreducible representations of $G$.

If $\gamma$ is a finite dimensional representation of $G$ we consider the homomorphism $L_{f} \rightarrow \int f(x) \gamma(x) d x$ of $a$ into operators on the representation space of $\gamma$. Since $G$ has a faithful finite dimensional representation, the Stone-Weierstrass theorem implies that these homomorphisms separate $Q$. Consequently, since $G$ is semi-simple, the ones arising from irreducible $\gamma$ separate $\alpha$. Let $Q=\int_{K} \chi(k) \gamma(k) d_{K} k$. Then $E L_{f} E \rightarrow Q\left(\int f(x) \gamma(x) d x\right) Q$ under the homomorphism. It is, therefore, sufficient to observe that the dimension $q$ of the range of the projection $Q$ is $\leqq n^{2}$. But this is a consequence of the fact that $\gamma \mid K$ is a cyclic representation, which we shall now show. By Lie's theorem there exists a vector $v$ such that $\gamma(s) v=\lambda(s) v$ for all $s$ in $S$ where $\lambda(s)$ is a scalar. Since $\gamma$ is irreducible $\gamma(G) v=\gamma(K) \gamma(S) v=\lambda(S) \gamma(K) v$ spans the representation space, and so $\gamma(K) v$ does also.

Corollary. Let $G$ be a connected semi-simple matrix group. Then every continuous unitary representation of $G$ is of type $I$. 
Proof. Let $U$ be a unitary representation of $G$, and let $\rho$ be any irreducible representation of a maximal compact subgroup $K$. Let $\bar{\chi}_{\rho} / n$ be the character of $\rho$ where $n$ is the degree of $\rho$. Let $P_{\rho}$ $=\int \chi_{\rho}(x) U(x) d x$. Let $u$ be the von Neumann algebra generated by $U$. Then $P_{\rho} u P_{\rho}$ is of type $I_{\leqq}{ }^{2}$ since a number of subalgebras of $E \& E$ have homomorphic images which are weakly dense in $P_{\rho} u P_{\rho}$. But $\left\{P_{\rho}\right\}$ is an orthogonal family of projections whose sum is $I$. Hence $u$ is of type $I$.

\section{BiBLIOGRAPHY}

1. R. Godement, $A$ theory of spherical functions I, Trans. Amer. Math. Soc. vol. 73 (1952) pp. 496-556.

2. - Thêorie des caractères II. Définition et propriétés générales des caractères, Ann. of Math. vol. 59 (1954) pp. 63-85.

3. Harish-Chandra, Representations of a semi-simple Lie group on a Banach space I, Trans. Amer. Math. Soc. vol. 75 (1953) pp. 185-243.

4. K. Iwasawa, On some types of topological groups, Ann. of Math. vol. 50 (1949) pp. 507-558.

5. I. Kaplansky, Groups with representations of bounded degree, Canad. J. Math. vol. (1949) pp. 105-112.

Institute for Advanced Study 